\title{
Health-related quality of life, depression, and self-esteem in adolescents with leprosy-affected parents: results of a cross-sectional study in Nepal
}

\author{
Nobuko Yamaguchi ${ }^{1}$, Krishna C Poudel ${ }^{2^{*}}$ and Masamine Jimba ${ }^{3}$
}

\begin{abstract}
Background: Leprosy is a chronic infectious disease that has an impact on the Health-Related Quality of Life (HRQOL) of sufferers as well as their children. To date, no study has investigated the effects of parental leprosy on the well-being of adolescent children.

Methods: A cross-sectional study was conducted in the Lalitpur and Kathmandu districts of Nepal. Adolescents with leprosy-affected parents ( $n=102$; aged $11-17$ years) and those with parents unaffected by leprosy $(n=115$; 11-17 years) were investigated. Self-reported data from adolescents were collected using the Kinder Lebensqualität Fragebogen $\left(K I N D L^{R}\right)$ questionnaire to assess HRQOL, the Center for Epidemiological Studies-Depression Scale (CESD), and the Rosenberg Self-esteem Scale (RSES). Analysis of covariance (ANCOVA) was used to compare scores between the two groups. Multiple regression analysis was conducted to explore the determinants of HRQOL for adolescents with leprosy-affected parents.
\end{abstract}

Results: ANCOVA revealed that the KINDL ${ }^{R}$ and RSES scores were significantly lower among adolescents with leprosy-affected parents compared with unaffected parents. However, the scores of "Friends" and "School" subscales of KINDL ${ }^{R}$ were similar between the two groups. The CES-D score was significantly higher among adolescents with leprosy-affected parents than for adolescents with unaffected parents. The KINDL ${ }^{\mathrm{R}}$ scores for adolescents with both parents affected $(n=41)$ were significantly lower than the scores for those with one parent affected $(n=61)$. Multiple regression analysis revealed that adolescents with leprosy-affected parents who had higher levels of depressive symptoms were more likely to have lower $\mathrm{KINDL}^{\mathrm{R}}$ scores. A similar result was seen for adolescents where both parents had leprosy.

Conclusions: Adolescents with leprosy-affected parents had higher levels of depressive symptoms, lower levels of self-esteem, and lower HRQOL compared with adolescents whose parents were unaffected by leprosy. Thus, mental health support programs might be necessary for adolescents with leprosy-affected parents, particularly for adolescents where both parents are leprosy-affected. Further studies with larger sample sizes are necessary to draw decisive conclusions.

Keywords: Adolescents, Leprosy-affected parents, Quality of life, Mental health, Nepal

\footnotetext{
*Correspondence: krishna@schoolph.umass.edu

2Department of Public Health, School of Public Health and Health Sciences, University of Massachusetts Amherst, 316 Arnold House, 715 North Pleasant St, Amherst, MA 01003-9304, USA

Full list of author information is available at the end of the article
} 


\section{Background}

Leprosy is a chronic infectious disease that typically affects the skin and peripheral nerves. According to the World Health Organization (WHO), the South East Asia region accounts for $59.2 \%$ of leprosy cases worldwide [1]. The WHO has developed a global strategy for 20112015 that aims to reduce the burden of leprosy, and improve treatment and early detection of new cases. For rehabilitation, patients need to be provided with highquality care to reduce the number of disabilities, and to deal with the socioeconomic stigma associated with leprosy [2].

Nepal is one of many South Asian countries where leprosy is endemic. Leprosy patients are stigmatized in Nepalese society, particularly those with visual deformities [3]. The family members of patients also experience limitations and restrictions in their social life $[4,5]$. Although intensive rehabilitation of psychological and socioeconomic support networks of leprosy-affected people and their families is increasing, these individuals have to deal with a poor quality of life and an ongoing struggle against stigma [6-8]. Nepalese people with a Hindu cultural background generally believe that leprosy is a result of ritually unclean behavior or a form of punishment for a misdeed (or misdeeds) in a former life [4]. However, public awareness of leprosy is increasing because of the spread of health services treating this affliction, improving literacy rates, and because of a change in attitude regarding traditional beliefs in some regions of Nepal [9].

Leprosy has been demonstrated to have an impact on Health-related Quality of Life (HRQOL) and the mental health status of affected individuals [10-12]. The selfesteem as well as physical and emotional well-being of leprosy patients and their families are likely to be affected. It has also been postulated that the school lives of adolescent children whose parents suffer from leprosy are also affected [13-16].

Parental diseases are known to influence the HRQOL or well-being of adolescents and children. Common diseases where this has been investigated include Parkinson's disease [17], multiple sclerosis [18], mental illness [19], and HIV/AIDS [20]. Similar to observations seen for other chronic diseases, the mental health of adolescent children, whose parents suffer from leprosy, can be adversely affected. However, very little research regarding this topic has been published. Previously, the importance of epidemiological studies has been emphasized for leprosy-affected children in endemic areas [21,22], but not for adolescents with leprosy-affected parents.

The objective of this study was to investigate the impact of parental leprosy on the well-being of adolescents. We hypothesized that HRQOL, depression, and selfesteem scores between adolescents with and without leprosy-affected parents would be different. Additionally, there might be an apparent difference in HRQOL, depression, and self-esteem scores between adolescents with one leprosy-affected parent and those with both parents affected. We also hypothesized that demographic characteristics, depression, and self-esteem would correlate with HRQOL scores for adolescents with leprosyaffected parents.

\section{Methods}

This was a cross-sectional study among adolescents with and without leprosy-affected parents. In this study, a leprosy-affected parent was defined as the parent of an adolescent that had previously undergone, or was currently undergoing, treatment for leprosy.

\section{Participants}

This study was conducted in the Lalitpur and Kathmandu districts of Nepal. Adolescents of leprosy-affected parents were recruited from the National Leprosarium of Lalitpur and local hostels for school-aged children. Only schoolage children with leprosy or leprosy-affected parents reside in these hostels. Most children in these hostels were from the National Leprosarium while others were from the Central Region of Nepal. Adolescents who live in hostels return to their homes on holidays. The leprosarium accommodates approximately 250 leprosy-affected people and their families. Institutional care in the leprosarium is confined to a basic supply of food, clothing, benefit money, and medical care [23].

The inclusion criterion in the present study was age between 11 and 17 years. The exclusion criteria for adolescents were: (i) having received a diagnosis of leprosy or any other chronic disease within 2 months prior to the fieldwork; and (ii) not willing to participate in the study.

In total, 135 participants with leprosy-affected parents and an equal number of adolescents with parents unaffected by leprosy as controls were selected. Informed consent sheets were distributed to all participants and parents' signatures were collected to verify parental consent. An adolescent was only included in the study if a parent of the adolescent provided written informed consent. Thirty-three adolescents with leprosy-affected parents and 20 adolescents without leprosy-affected parents were excluded, as they were not willing to participate in this study. Eventually, 102 adolescents of leprosy-affected parents and 115 adolescents with parents unaffected by leprosy were enrolled in this study. Of the 102 adolescents of leprosy-affected parents, 78 were recruited from hostels and 24 from the leprosarium. Adolescents of leprosyunaffected parents were recruited from two schools in the study area. The education system of Nepal consists of 5 years of primary education, 3 years of lower secondary 
education, 2 years of secondary education, and 2 years of higher secondary education.

The study protocol was approved by the Ethical Committees of The University of Tokyo, Japan, and the Nepal Health Research Council, Nepal.

\section{Measures}

Measures included demographic characteristics and assessments of HRQOL, depression, and self-esteem. HRQOL was assessed using the Kinder Lebensqualität Fragebogen $\left(\mathrm{KINDL}^{\mathrm{R}}\right)$, which covers "Physical WellBeing", "Emotional Well-Being", "Self-Esteem", "Family", "Friends", and "School". The psychometric results of this scale have a high reliability and validity among children and adolescents using various languages $[24,25]$. KINDL $^{\mathrm{R}}$ offers 24 items referring to the past week with 5-point Likert-scales ("Never" to "All the time"), with 11 items being reverse-coded. The scores range from 0 to 100 , where higher scores indicate higher levels of HRQOL among teenage adolescents. A Nepalese version of $\mathrm{KINDL}^{\mathrm{R}}$ has internal consistency, reproducibility, responsiveness, interpretability, and discriminant validity [26].

The depressive status of participants was measured using the Center for Epidemiological Studies-Depression Scale (CES-D). This scale is one of the most frequently used standardized measurements in primary depression screening, and is widely used for clinical and research purposes among general populations [27]. CES-D offers 20 items with 4-point Likert-scales ("Rarely or none of the time" to "Most or all of the time"), with 4 items being reverse-coded. For each statement, the subject chooses the response that best describes how often they felt or behaved this way during the past week. The scores range from 0 to 60 , with higher scores indicating higher levels of depressive mood.

Self-esteem was examined using the Rosenberg SelfEsteem Scale (RSES). Globally, RSES is the most widely used scale to measure self-esteem among adolescents [28]. RSES offers 10 items with 4-point Likert-scales ("Strongly agree" to "Strongly disagree"), with 5 items being reverse-coded. Participants rated the extent to which they have experienced each symptom over the past week. The scores range from 0 to 30 , with higher scores indicating higher levels of self-esteem.

\section{Data collection}

Data were collected from March to May 2008 using the self-administered questionnaire survey. All participants completed the questionnaires by themselves. Data were collected from the Leprosarium by conducting a door-todoor survey based on a resident's list. The questionnaire survey was carried out at the hostels and data collected during the school breaks. In schools, the questionnaires were administered after school. All participants answered the questionnaire without the intervention of their guardian or teachers.

\section{Data analysis}

The demographics between adolescents with and without leprosy-affected parents were compared. Significant differences of categorical variables [gender, category (years), and school grade] and of continuous variables of age were tested by Chi-square tests and $t$-tests, respectively. The score distributions of KINDL ${ }^{\mathrm{R}}, \mathrm{CES}-\mathrm{D}$, and RSES between adolescents with and without leprosy-affected parents were measured and compared between the two groups. The analysis of covariance (ANCOVA) was used with grade as a factor, since a significant difference in this variable was detected. Grade was a dichotomous variable with two values (1-5, 6-12). For adolescents with leprosyaffected parents, the total and the subscale scores of KINDL $^{\mathrm{R}}$, CES-D, and RSES were compared between adolescents having one parent or both parents affected with leprosy. The $t$-test was performed for the differences between the mean values of these scores.

Cohen's effect size was used, taking the differences between the two means divided by the standard deviation of the scores. Effect sizes were interpreted as small (0.2), medium (0.5), and large (above 0.8) [29].

To explore the determinants of HRQOL for adolescents with leprosy-affected parents, multiple regression analysis with the total score of $\mathrm{KINDL}^{\mathrm{R}}$ as a dependent variable were conducted. Age, gender, parental status (one or both parents affected with leprosy), residence of participants (leprosarium or hostels), and CES-D total scores were selected as independent variables. RSES scores and grade were excluded from the model because of the multicollinearity with CES-D and age (Variance Inflation Factor [VIF]: > 2.0), respectively. All statistical analyses were performed using SPSS ver.16.0 (SPSS Inc., Chicago, IL).

\section{Results}

Table 1 shows the demographic characteristics of the study participants. The mean ages of 102 adolescents with leprosy-affected parents and 115 adolescents without leprosy-affected parents were 13.8 years and 14.5 years, respectively. Of the total, $36.3 \%$ of adolescents of parents affected with leprosy were in primary school while $63.7 \%$ were in secondary school. Significant differences in mean age, age category (years old), and grade were identified between these two groups.

Among adolescents with leprosy-affected parents, 78 were living in hostels and 24 were cohabiting with parents in the leprosarium. Among these participants, 61 adolescents had one parent with a history of leprosy (father; 53, mother; 8) and 41 had two parents with a history of leprosy. 
Table 1 Demographic characteristics of the participants

\begin{tabular}{|c|c|c|c|c|c|}
\hline & \multicolumn{2}{|c|}{$\begin{array}{l}\text { Adolescents with parents affected } \\
\text { by leprosy }{ }^{1}(n=102)\end{array}$} & \multicolumn{2}{|c|}{$\begin{array}{l}\text { Adolescents with parents unaffected } \\
\text { by leprosy }{ }^{2)}(n=115)\end{array}$} & \multirow[t]{2}{*}{$\begin{array}{c}p \\
\text { value }^{3}\end{array}$} \\
\hline & $\mathbf{n}$ & (\%) & $\mathbf{n}$ & (\%) & \\
\hline \multicolumn{6}{|l|}{ Gender } \\
\hline Boys & 50 & $(49.0)$ & 56 & $(48.7)$ & .962 \\
\hline Girls & 52 & $(51.0)$ & 59 & $(51.3)$ & \\
\hline \multicolumn{6}{|l|}{ Age } \\
\hline Mean (SD) & 13.8 & (1.6) & 14.5 & (1.3) & .002 \\
\hline \multicolumn{6}{|l|}{ Category (years) } \\
\hline 11 & 6 & $(5.9)$ & 3 & $(2.6)$ & .003 \\
\hline 12 & 23 & $(22.5)$ & 6 & $(5.2)$ & \\
\hline 13 & 15 & $(14.7)$ & 15 & $(13.0)$ & \\
\hline 14 & 21 & $(20.6)$ & 30 & $(26.1)$ & \\
\hline 15 & 17 & $(16.7)$ & 37 & $(32.2)$ & \\
\hline 16 & 15 & $(14.7)$ & 19 & $(16.5)$ & \\
\hline 17 & 5 & $(4.9)$ & 5 & $(4.3)$ & \\
\hline \multicolumn{6}{|l|}{ School grade ${ }^{4)}$} \\
\hline $1-5$ & 37 & $(36.3)$ & 0 & $(0.0)$ & $<.001$ \\
\hline $6-12$ & 65 & $(63.7)$ & 115 & $(100.0)$ & \\
\hline \multicolumn{6}{|l|}{ Residence of participants } \\
\hline Hostel & 78 & $(76.5)$ & & & \\
\hline Leprosarium & 24 & $(23.5)$ & & & \\
\hline \multicolumn{6}{|c|}{ Participants with one or both parents affected by leprosy } \\
\hline One parent & 61 & $(59.8)$ & & & \\
\hline Both parents & 41 & $(40.2)$ & & & \\
\hline
\end{tabular}

Table 2 shows the total and subscale scores and comparative test results for $\mathrm{KINDL}^{\mathrm{R}}$, CES-D, and RSES scores of adolescents with and without leprosy-affected parents. The means of total KINDL ${ }^{\mathrm{R}}$ scores among adolescents with leprosy-affected parents and those without were 55.9 (SD 13.1) and 63.0 (SD 9.9), respectively. The effect size was medium (0.63) in magnitude between the two groups. Subscale scores were significantly lower among the participants with leprosy-affected parents compared with those without except for the subscales of "Friends" and "School". The "Self-Esteem" subscale had the lowest score and "Friends" subscale had the highest score among adolescents with leprosy-affected parents. The lowest and highest subscales in KINDL $^{\mathrm{R}}$ among adolescents with parents unaffected by leprosy were "School" and "Family", respectively. The effect sizes of subscales between the two groups were large to small (1.04 to 0.06).

The means of the total scores of CES-D and RSES were compared between both groups. The CES-D score among adolescents with leprosy-affected parents was significantly higher than for adolescents without leprosyaffected parents. The mean of the total RSES score was significantly lower among adolescents with leprosyaffected parents than among those without leprosyaffected parents. Cronbach's alpha values of $\mathrm{KINDL}^{\mathrm{R}}$, CES-D, and RSES were $0.81,0.80$, and 0.70 , respectively.

Table 3 shows comparisons of KINDL ${ }^{R}, C E S-D$, and RSES scores in adolescents with one parent and both parents affected with leprosy. A $t$-test was performed to determine the mean difference between the two groups owing to smaller sample sizes. Means of total scores of $\mathrm{KINDL}^{\mathrm{R}}$ for adolescents with one parent or both parents affected with leprosy were 59.6 (SD 11.0) and 50.4 (SD 14.0), respectively. Overall, the subscale scores of adolescents with both parents affected were significantly lower than for those with one parent affected, except for the "Physical Well-Being" subscale. The effect sizes between the two groups were medium to small (0.74 to 0.11 ). 
Table 2 Comparison of the KINDL ${ }^{\mathrm{R},}$ CES-D, and RSES scores for adolescents with leprosy-affected parents and adolescents with parents unaffected by leprosy

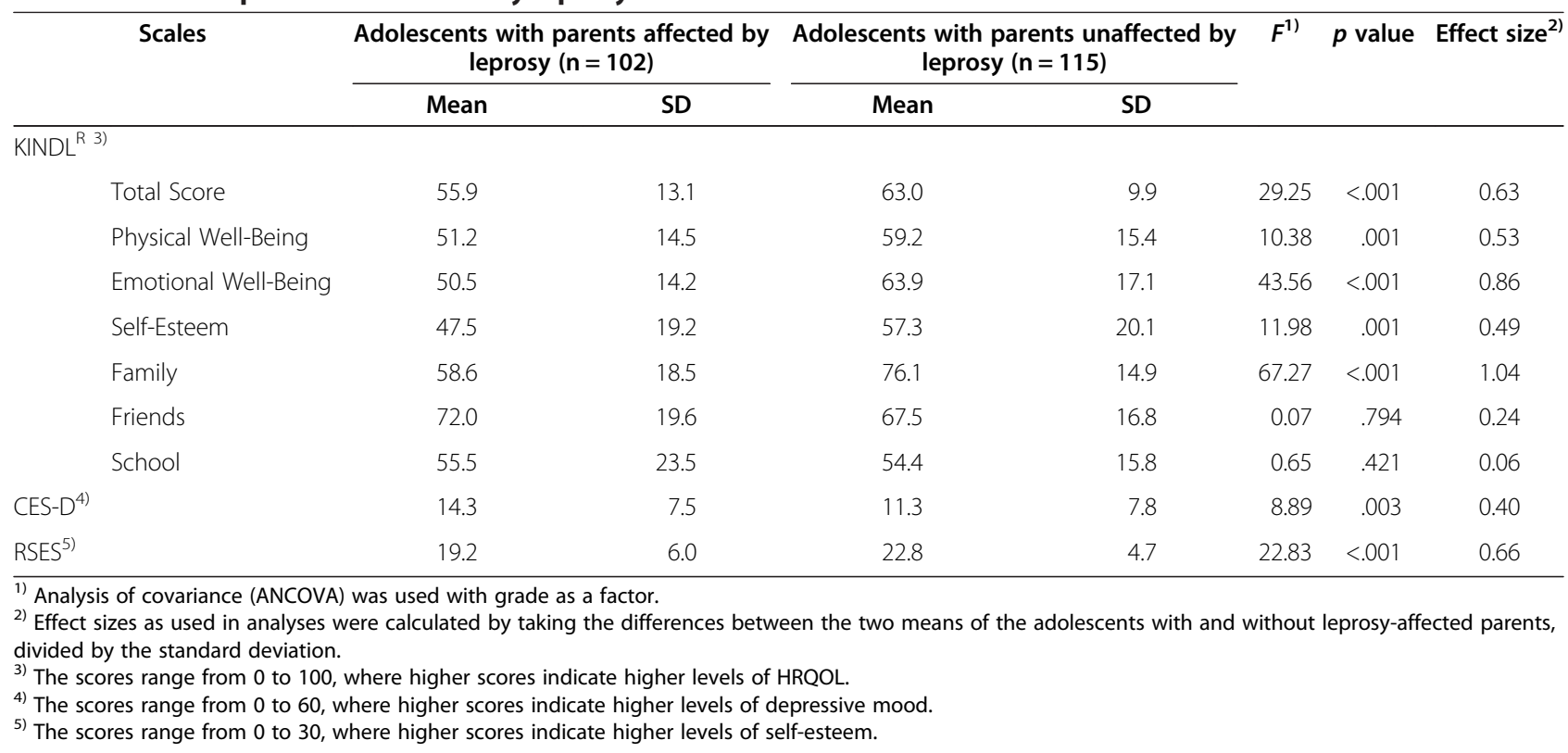

CES-D and RSES showed no significant differences between the two subgroups.

Multiple regression analysis was conducted to identify determinants of HRQOL (Table 4). Adolescents with higher levels of depressive symptoms and those having two leprosy-affected parents were more likely to have lower $\mathrm{KINDL}^{\mathrm{R}}$ scores. In the multivariable regression analysis, the value for adjusted R-Squared was 0.44 . The value of R-Squared is a quantitative measure of how well the independent variables account for the outcome. In this study, CES-D and parental leprosy status were more important HRQOL predictors for adolescents with leprosy-affected parents.

\section{Discussion}

The aim of the present study was to investigate the impact of parental leprosy on the well-being of adolescent children. The study found that adolescents with leprosyaffected parents had higher levels of depressive symptoms and lower levels of self-esteem and HRQOL. Our results have important implications for implementing mental health programs for adolescents with leprosy-

Table 3 Comparison of the KINDL ${ }^{\mathrm{R},}$ CES-D, and RSES scores for adolescents with one and both parents affected by leprosy

\begin{tabular}{|c|c|c|c|c|c|c|c|}
\hline & \multirow[t]{2}{*}{ Scales } & \multicolumn{2}{|c|}{$\begin{array}{l}\text { Adolescents with one parent } \\
\text { affected by leprosy }(n=61)\end{array}$} & \multicolumn{2}{|c|}{$\begin{array}{l}\text { Adolescents with both parents } \\
\text { affected by leprosy }(n=41)\end{array}$} & \multirow[t]{2}{*}{$\begin{array}{c}p \\
\text { value }^{1)}\end{array}$} & \multirow[t]{2}{*}{$\begin{array}{l}\text { Effect } \\
\text { size }^{2)}\end{array}$} \\
\hline & & Mean & SD & Mean & SD & & \\
\hline \multicolumn{8}{|l|}{$\mathrm{KINDL}^{\mathrm{R}}$} \\
\hline & Total Score & 59.6 & 11.0 & 50.4 & 14.0 & $<.001$ & 0.74 \\
\hline & Physical Well-Being & 51.9 & 15.0 & 50.3 & 13.8 & .602 & 0.11 \\
\hline & Emotional Well-Being & 53.1 & 12.9 & 46.7 & 15.4 & .024 & 0.45 \\
\hline & Self-Esteem & 51.8 & 18.1 & 41.2 & 19.3 & .006 & 0.57 \\
\hline & Family & 62.4 & 15.6 & 52.9 & 21.2 & .010 & 0.52 \\
\hline & Friends & 77.3 & 16.5 & 64.0 & 21.2 & .001 & 0.70 \\
\hline & School & 61.1 & 21.2 & 47.1 & 24.5 & .003 & 0.61 \\
\hline CES-D & & 13.6 & 7.6 & 15.4 & 7.2 & .229 & 0.25 \\
\hline RSES & & 19.7 & 5.9 & 18.5 & 6.3 & .345 & 0.19 \\
\hline
\end{tabular}

1) $t$-test was used for significant differences between adolescents with one and both parents affected by leprosy.

${ }^{2}$ Effect sizes as used in the analyses were calculated by taking the differences between the two means of the adolescents with one and both parents affected by leprosy, divided by the standard deviation. 
Table 4 Multiple regression analysis of the $\mathrm{KINDL}^{\mathrm{R}}$ among adolescents with leprosy-affected parents $(n=102)$

\begin{tabular}{|c|c|c|c|}
\hline \multirow[t]{2}{*}{ Variables } & \multirow{2}{*}{$\frac{\mathrm{KINDL}^{\mathrm{R}} \text { total scores }}{\beta^{1)}}$} & \multicolumn{2}{|c|}{ 95\% Confidence Interval } \\
\hline & & Lower & Upper \\
\hline Age & 0.07 & -0.91 & 2.12 \\
\hline Gender ${ }^{2)}$ & -0.06 & -5.41 & 2.18 \\
\hline Participants with one or both parents affected by leprosy ${ }^{3)}$ & $-0.29^{* *}$ & -11.39 & -3.75 \\
\hline Residence of participants ${ }^{4)}$ & -0.07 & -6.65 & 2.20 \\
\hline CES-D & $-0.54^{* *}$ & -1.21 & -0.69 \\
\hline
\end{tabular}

1) Standard multiple regression analysis was used to explore the determinants of the HRQOL for the adolescents with leprosy-affected parents. R-Squared $=0.46$, adjusted R-Squared $=0.44,^{* *} p<.001$.

2) Gender ( 0 = girls, 1 = boys).

3) Participants with one or both parents affected by leprosy ( $0=$ one parent , $1=$ both parents).

${ }^{4)}$ Residence of participants $(0=$ leprosarium, $1=$ hostel).

affected parents who currently have limited access to such programs.

In this study, one of the inclusion criteria of adolescents with leprosy-affected parents was not having chronic diseases. The "Physical Well-Being" score of the $\mathrm{KINDL}^{\mathrm{R}}$ was lower for adolescents with leprosy-affected parents than for those with unaffected parents. Adolescents with leprosy-affected parents are vulnerable to the onset of leprosy [30] and thus, they might worry about contracting the disease despite being in good health and having regular health check-ups.

Adolescents with leprosy-affected parents had lower "Emotional Well-Being" and "Self-Esteem" scores than those of general adolescents. Self-esteem is strongly related to emotional well-being and is an emotional component of personal qualities and competencies. It is generally related to how well or poorly individuals feel about themselves [28]. Leprosy-affected people and their family members are often excluded from social participation at the community level [4,5,7-9]. Such experiences of adolescents with leprosy-affected parents might contribute to their low self-esteem and poor emotional state. Over $90 \%$ of students in public schools in the study area come from the poorest quintiles in Nepal [31]. Thus, low self-esteem of the participants in this study might be due to the poverty of their families. Adolescents are highly susceptible to the impact of family events such as poverty, unemployment, and other adverse social circumstances [32].

The KINDL ${ }^{\mathrm{R}}$ scores might vary according to these participants' background. Many adolescents of leprosyaffected parents cannot go to school because of the poor state of the parents' economic situation [15]. Thus, there is a need to deal with such issues and further enhance the educational environment for these adolescents.

The "Friends" and "School" subscale scores were not significantly different between adolescents with and without leprosy-affected parents. In the hostels or the leprosarium, adolescents stay with other primary stakeholders of leprosy. It is unlikely that the adolescents are discriminated against by adolescents of a similar status. A previous study [33] suggested that the partnerships formed within minority groups promote the strength of solidarity. In addition, fieldwork for this study was conducted soon after school final examinations. In Nepal, such examinations are generally stressful because students might fail and be required to repeat the same grade [34]. This situation might have contributed to the low levels of school subscale scores among the studied adolescents.

Mental health studies in developed and developing countries show that between $10-25 \%$ of children and adolescents suffer from a mental health problem [35]. The high prevalence of depressive symptoms among adolescents with parents unaffected by leprosy in this study might be due to their economic backgrounds. Mental health programs are difficult to access for most of the population in Nepal.

The KINDL ${ }^{\mathrm{R}}$ total and subscale scores of adolescents with two parents affected with leprosy were significantly different from those with only one parent affected. In addition, the presence of depression and having two parents affected with leprosy significantly affected adolescents' HRQOL. A previous study reported a similar result where the risk of mental health problems for adolescents was greater when both parents had mental health problems than when a single parent had a mental health problem [36]. Adolescents with leprosy-affected parents might be involved in daily household activities including caring for family members as they grow up [17], especially when both parents are affected with leprosy-related disabilities. Thus, the burden of leprosy and related social problems might be more severe among adolescents with two parents affected with leprosy compared with those with only one parent affected. The results of the present study may be helpful in evaluating the environment of adolescents with leprosyaffected family members and for assessing the range of interventions that might be appropriate.

Our results suggest the need for implementing mental health programs for adolescents with leprosy-affected parents, in particular, those adolescents with both parents 
affected with leprosy. Moreover, the programs should be designed to reduce or prevent stigma among adolescents with leprosy-affected parents. Most of the patients and their family members, as primary stakeholders in leprosy, are also vulnerable to public stigmatization and misinformation that causes fear or anxiety. Such programs should aim to reduce depressive symptoms and improve selfesteem and subjective well-being among adolescents with leprosy-affected parents, and help them to cope with their parents' disease [37].

One limitation of our study is the small sample size, and it may not be possible to generalize our results to all adolescents with leprosy-affected parents. Thus, the suggested interventions may not be definitive in Nepal or other countries. However, it is difficult to recruit sufficient participants to adequately represent the target population, because home-based treatment of leprosy patients is common in Nepal as patients want to conceal their disease from the local community. Nevertheless, we recruited participants residing either in the leprosarium or in hostels. The response rate of participants in the study was $75 \%$ $(102 / 135)$. Therefore, our results are applicable to adolescents residing in similar institutions.

Our study has another limitation. The CES-D is a selfrating instrument to identify depressive symptoms during the previous week, and is not a diagnostic tool to identify depression administered by a suitably trained professional.

\section{Conclusions}

Adolescents with leprosy-affected parents had higher levels of depressive symptoms and lower levels of self-esteem and HRQOL compared with those without leprosyaffected parents. Further study with a larger sample size is necessary to confirm our conclusions. Our results suggest that mental health support programs are necessary for adolescents with leprosy-affected parents, in particular, for adolescents with two parents affected with leprosy.

\section{Abbreviations \\ HRQOL: Health-related Quality of Life; KINDL: Kinder Lebensqualität Fragebogen; CES-D: Center for Epidemiological Studies-Depression Scale; RSES: Rosenberg Self-esteem Scale.}

\section{Competing interests}

The study was supported by Saitama Prefectural University, Saitama, Japan. The authors declare that they have no conflict of interest.

\section{Authors' contributions}

NY conducted the survey and drafted the initial manuscript. KCP and MJ supervised the study. KCP and MJ helped in the statistical analysis and with manuscript revision. All the authors have read and approved this submission.

\section{Acknowledgments}

We would like to thank all the adolescents for their participation in this study. We also thank the guardians, school teachers, Mr. Hera Kaji Shakya, and Mr. Bidur Basnet of the Nepal Leprosy Relief Association for their cooperation and support. We are also grateful to Dr. Cheng-Fang Yen and
Dr. Ulrike Ravens-Sieberer for their constructive comments to improve our manuscript.

\section{Author details}

${ }^{1}$ Department of Nursing, School of Health and Social Services, Saitama Prefectural University, 820 Sannomiya, Koshigaya, Saitama 343-8540, Japan. ${ }^{2}$ Department of Public Health, School of Public Health and Health Sciences, University of Massachusetts Amherst, 316 Arnold House, 715 North Pleasant St, Amherst, MA 01003-9304, USA. ${ }^{3}$ Department of Community and Global Health, Graduate School of Medicine, The University of Tokyo, 7-3-1 Hongo, Bunkyo-ku, Tokyo 113-0033, Japan.

Received: 21 May 2012 Accepted: 23 December 2012

Published: 10 January 2013

\section{References}

1. World Health Organization: Leprosy update, 2011. Wkly Epidemiol Rec 2011, 86:389-400

2. World Health Organization: Global leprosy situation, 2010. Wkly Epidemiol $\operatorname{Rec} 2010,85: 337-348$.

3. Van Brakel WH, Anderson AM, Mutatkar RK, Bakirtzief Z, Nicholls PG, Raju MS, Das-Pattanayak RK: The Participation Scale: measuring a key concept in public health. Disabil Rehabil 2006, 28:193-203.

4. de Stigter DH, de Geus L, Heynders ML: Leprosy: between acceptance and segregation. Community behavior towards persons affected by in eastern Nepal. Lepr Rev 2000, 71:492-498.

5. Nicholls PG, Bakirtzief Z, Van Brakel WH, Das-Pattanaya RK, Raju MS, Norman G, Mutatkar RK: Risk factors for participation restriction in leprosy and development of a screening tool to identify individuals at risk. Lepr Rev 2005, 76:305-315.

6. Brandsma JW, Harijan T, Wagle P, Baxter KA: Partnership for rehabilitation: looking beyond impairments. Indian J Lepr 2008, 80:19-29.

7. Cross H, Choudhary R: STEP: an intervention to address the issue of stigma related to leprosy in Southern Nepal. Lepr Rev 2005, 76:316-324.

8. Heijnders ML: The dynamics of stigma in leprosy. Int J Lepr Other Mycobact Dis 2004, 72:437-447.

9. Varkevisser CM, Lever P, Alubo O, Burathoki K, Idawani C, Moreira TM, Patrobas P, Yulizar M: Gender and leprosy: case studies in Indonesia, Nigeria, Nepal and Brazil. Lepr Rev 2009, 80:65-76.

10. Mankar MJ, Joshi SM, Velankar DH, Mhatre RK, Nalgundwar AN: A Comparative study of the quality of life, knowledge, attitude and belief about leprosy disease among leprosy patients and community members in Shantivan Leprosy Rehabilitation Centre, Nere, Maharashtra, India. J Glob Infect Dis 2011, 3:378-382.

11. Lustosa AA, Nogueira LT, Pedrosa JI, Teles JB, Campelo V: The impact of leprosy on health-related quality of life. Rev Soc Bras Med Trop 2011, 44:621-626.

12. Tsutsumi A, Izutsu T, Islam AM, Maksuda AN, Kato H, Wakai S: The quality of life, mental health, and perceived stigma of leprosy patients in Bangladesh. Soc Sci Med 2007, 64:2433-2453.

13. Ebenso B, Ayuba M: 'Money is the vehicle of interaction': Insight into social integration of people affected by leprosy in northern Nigeria. Lepr Rev 2010, 81:99-110.

14. Scott J: The psychosocial needs of leprosy patients. Lepr Rev 2000, 71:486-491.

15. Saylan T, Karadeniz A, Iyier N, Soydan M, Pamuk D: A scholarship project for the children of leprosy patients in Turkey. Lepr Rev 2000, 71:212-216.

16. Kopparty SNM, Kurup AM, Sivaram M: Problems and coping strategies of families having patients with and without deformities. Indian J Lepr 1995, 67:133-152.

17. Schrag A, Morley D, Quinn N, Jahanshahi M: Impact of Parkinson's disease on patients' adolescent and adult children. Parkinsonism Relat Disord 2004, 10:391-397.

18. Morley D, Selai C, Schrag A, Jahanshahi M, Thompson A: Adolescent and adult children of parents with Parkinson's disease: incorporating their needs in clinical guidelines. Parkinsons Dis 2011, 2011:951874. Epub 2011 Jun 12.

19. Giannakopoulos G, Dimitrakaki C, Pedeli X, Kolaitis G, Rotsika V, RavensSieberer U, Tountas Y: Adolescents' wellbeing and functioning: relationships with parents' subjective general physical and mental health. Health Qual Life Outcomes 2009, 7:100. doi:10.1186/1477-7525-7-100. 
20. Xu T, Wu Z, Rou K, Duan S, Wang H: Quality of life of children living in HIV/AIDS-affected families in rural areas in Yunnan, China. AIDS Care 2010, 22:390-396.

21. Sachdeva S, Amin SS, Khan Z, Sharma PK, Bansal S: Childhood leprosy: lest we forget. Trop Doct 2011, 41:163-165.

22. Madarasingha NP, Senaviratne JK: A study of household contacts of children with leprosy. Ceylon Med J 2011, 56:112-114.

23. Malla OK, Brandt F, Anten JGF: Ocular findings in leprosy patients in an institution in Nepal (Khokana). Br J Ophthalmol 1981, 65:226-230.

24. Helseth S, Lund T, Christophersen KA: Health-related quality of life in a Norwegian sample of healthy adolescents: some psychometric properties of CHQ-CF87-N in relation to KINDL-N. J Adolesc Health 2006, 38:416-425.

25. Ravens-Sieberer $U$, Bullinger M: Assessing health-related quality of life in chronically ill children with the German KINDL: first psychometric and content analytical results. Qual Life Res 1998, 7:399-407.

26. Yamaguchi N, Poudel KC, Poudel-Tandukar K, Shakya D, Ravens-Sieberer U, Jimba M: Reliability and validity of a Nepalese version of the KiddoKINDL in adolescents. Biosci Trends 2010, 44:178-185.

27. Radloff LS: The CES-D scale: a self report depression scale for research in the general population. Appl Psychol Meas 1977, 1:385-401.

28. Rosenberg M: Society and the adolescent self-image. Middletown, Connecticut: Wesleyan University Press; 1989.

29. Cohen J: Statistical power analysis for the behavioral sciences. 2nd edition. Hillsdale, NJ: Lawrence Earlbaum Associates; 1988.

30. Rao AG: Study of leprosy in children. Indian J Lepr 2009, 81:195-197.

31. Nepal Central Bureau of Statistics: Nepal living standard survey 2003/2004. Kathmandu: Nepal Central Bureau of Statistics; 2004.

32. Goodyear IM: Developmental psychopathology: the impact of recent life events in anxious and depressed school-age children. J R Soc Med 1994 87:327-329.

33. Nelson G, Prilleltensky I, MacGillivary H: Building value-based partnerships: toward solidarity with oppressed groups. Am J Community Psychol 2001, 29:649-677.

34. Nepal South Asia Center: Nepal human development report 1998. Kathmandu: Nepal South Asia Center; 1999.

35. World Health Organization: World Health Report 2001: Mental health: New understanding, new hope. Geneva: World Health Organization; 2001.

36. Quinton D, Rutter M: Family pathology and child psychiatric disorder. In Longitudinal Studies in Child Psychology and Psychiatry: Practical Lessons from research experience. Edited by Nicol AR. Chichester: Wiley; 1985:91-134.

37. Rotheram-Borus MJ, Lee MB, Gwadz M, Draimin B: An intervention for parents with AIDS and their adolescent children. Am J Public Health 2001, 91:1294-1302.

doi:10.1186/1471-2458-13-22

Cite this article as: Yamaguchi et al.: Health-related quality of life, depression, and self-esteem in adolescents with leprosy-affected parents: results of a cross-sectional study in Nepal. BMC Public Health 2013 13:22

\section{Submit your next manuscript to BioMed Central and take full advantage of:}

- Convenient online submission

- Thorough peer review

- No space constraints or color figure charges

- Immediate publication on acceptance

- Inclusion in PubMed, CAS, Scopus and Google Scholar

- Research which is freely available for redistribution 